\title{
Industrial Safety and Light Energy Optimization using FPGA
}

\author{
Manisha B. Kamthe \\ ME(E\&TC) Ilyr Student \\ Department of Electronics and Telecommunication \\ G.H.Raisoni College of Engineering and \\ Management Pune-India
}

\author{
Pravin N. Matte \\ Assistant Professor \\ Department of Electronics and Telecommunication \\ G.H.Raisoni College of Engineering and \\ Management Pune-India
}

\begin{abstract}
Industrial safety needs to pay more attention as there is risk of human life in most of the industrial places. So there should be less human intervention.For this purpose we need to automate the industrial processes.Industrial engineers need a more flexible development infrastructure. The structure should be such that it has ability to build in more function with higher processing speeds.It should be versatile.It should be such that the cost of infrastructure should be less.Onther aspect of this paper is to present the idea of light energy optimization.This is achieved by automatically controlling the streetlights.In this paper we present an idea of industrial automation and streetlight illumination by using FPGA.The proposed idea helps for rapid detection of dangerous situations.It uses twenty four hour real time monitoring and remote control of industrial devices.The system is based on GSM (Global System for Mobile). It sends a SMS (Short Messege Servicing) to the operator in case of emergency.The design has been described using FPGA.FPGAs are used as they are flexible than other processors available.Also they can be configured on field.The cost is also less.It uses different sensors for monitoring physical parameters such as temperature, $\mathrm{CO} 2$ etc.For controlling the streetlights we are using IR sensors and LDR. We are also using Bluetooth technology for in room operation.
\end{abstract}

\section{General Terms}

Energy optimization,Streetlight illumination,Industrial automation.

\section{Keywords}

FPGA, GSM, SMS, IRSensors,LDR,,Bluetooth,Remote control

\section{INTRODUCTION}

Safety in general means taking precautions of ourselves or of the system from the outside world so that it will not harm living being or that particular system.For example in home we make the gas off before our bread burns. Similar is the case in industry .A particular device should be closed if there is any possibility of damage to it e.g. increase in temperature or possibility of fire.For a person going in this particular situation is harmful for him as it may cause him physical or mental damage. With the advent of new technology it is possible to detect such dangerous situations and take the necessary preventive action remotely without going in that particular area. For this purpose we need to design a system which will monitor all the required parameters ,process it and take the necessary action such as closing a particular device.Also it is equally important to inform the person in charge so that he will take further necessary action.For monitoring different parameters we use analog sensors. This data is converted in digital form with the help of digital ICs like ADCs.Processing of the data will be taken care with the available processors such as RISC or CISC.We can also use microcontrollers such as the family of 51 series or FPGAs such as Spartan-3,6 or 7 series.[1][2]Along with this another factor comes into picture is the flexibility,cost competiveness and ease of deployment.Onther aspect also need more attention these days is energy conservation.Nothing happens without electricity these days.But the need of electricity is more than its available sources. So it is required to find the ways to reduce its consumption. The electric power required for glowing the street lamps is more.Most commonly used technique is using LDR where intensity of lamps will increase as darkness increases.But in this technique also not much of power is saved.New technique available these days is using IR sensors.Lamp will glow where human presence is detected.The aim of this paper is to present the idea of a device which is cost effective and control the industrial devices remotely as well as control the streetlights in the campus of industry. The device will use the GSM technology to send the SMS to operator.Bluetooth technology is used for in room operation of device. The heart of the device is FPGA.FPGA is used as it is cost effective,flexible and easily programmable.[7][8]

\section{RELATED WORK}

Earlier system used for industrial safety as well as security uses microprocessors like ARM or microcontrollers like PIC to control the automation.[3][4] These systems also use web technology.Existing systems also use programmable logic arrays i.e PLC to run the processes in the industry.Onther system is SCADA which is used for reading temperature and pressure which are common analog parameters in the process industry.Similarly existing systems for streetlight illumination uses LDR sensors, as the darkness increases intensity of light increases.But this system is also of little use in case of power saving.In our proposed idea we use FPGA as main controller.FPGA is used which saves cost,power and maintenance of the system.Also GSM technology is used for remote communication.Short Messege Service(SMS) is used for sending the messege to the operator.Bluetooth service is used for in room operation.In case of light optimization we use PIR sensors.These IR sensors senses the human presence[9][10].This information is given to processor and accordingly only that lamp post which is near to a particular vehicle will be glown.Thus a lot of energy is saved.Also due to industrial automation minimum human presence is needed in the vicinity of process plant. 


\section{PROPOSED ARCHITECTURE}

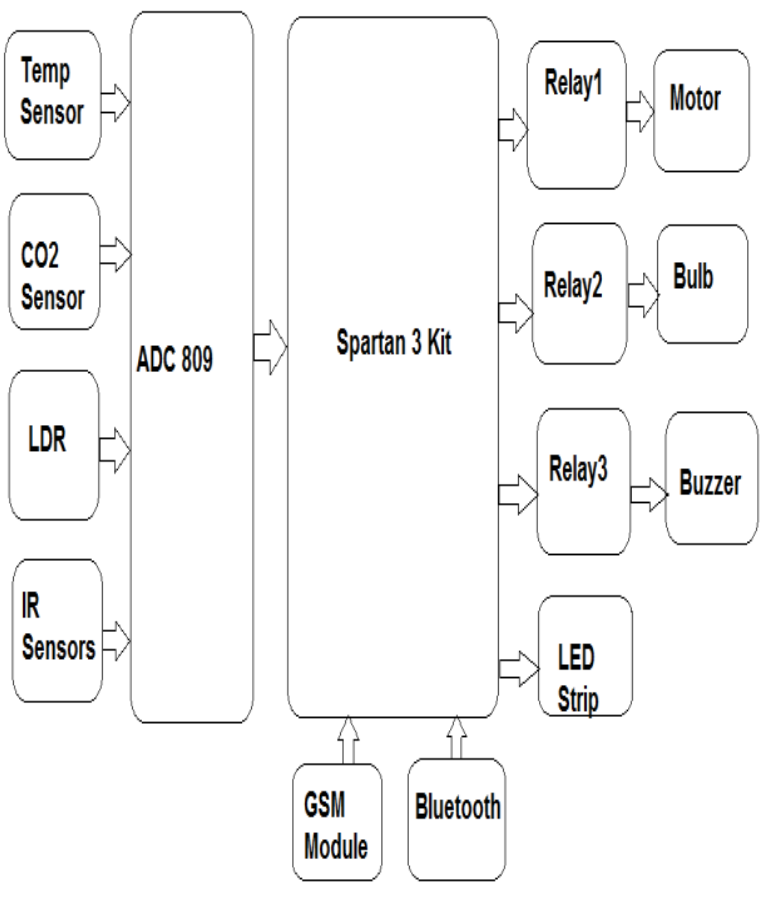

Fig 1: Proposed FPGA based System

As shown in the block diagram we are going to sense the three parameters here temperature,smoke(CO2) and light.The sensors used to sense the temperature is LM 35.The presence of $\mathrm{CO}$ 2(smoke) is sensed using sensor MQ7.[3][4]LDR is used to sense the intensity of light.IR sensors are used to sense the human presence.These analog parameters are converted into digital form.This is achieved with the help of ADC 809. The output of ADC is given to the FPGA board for processing. The FPGA kit chosen here is Spartan-3.The chip used is XC3S50AN.The output of the kit is given to SPDT relay which are used to turn ON or OFF the devices.GSM module is used to send the SMS for remote communication.It also uses Bluetooth for in room operation.[6][4]

\section{VARIOUS SYSTEM COMPONENTS}

\subsection{Temperature Sensor LM 35}

LM 35 is commonly used precision temperature sensor.The temperature range it senses is $-55 \geq^{0} \mathrm{C}$ to $+150^{\circ} \mathrm{C}$.It gives $10 \mathrm{mv}$ change for every degree of rise and fall in temperature.It draws only $60 \mu \mathrm{A}$ from its supply.It has very low self heating less than $0.1^{\circ} \mathrm{C}$ in air.[1]

\subsection{Smoke Sensor}

MQ7 is a carbon monoxide detector which detects the presence of $\mathrm{CO}$ in smoke in case of fire.The sensitivity may be adjusted by the potentiometer.[1]

\subsection{LDR}

LDR i.e. Light dependent resistor is used to sense the intensity of light.The output of LDR is used to control the intensity of streetlight.[9]

\subsection{PIR Sensors}

These IR sensors are used to detect the presence of humanbeing. These are simply sensitive to the infrared energy emitted by every living thing. [9]

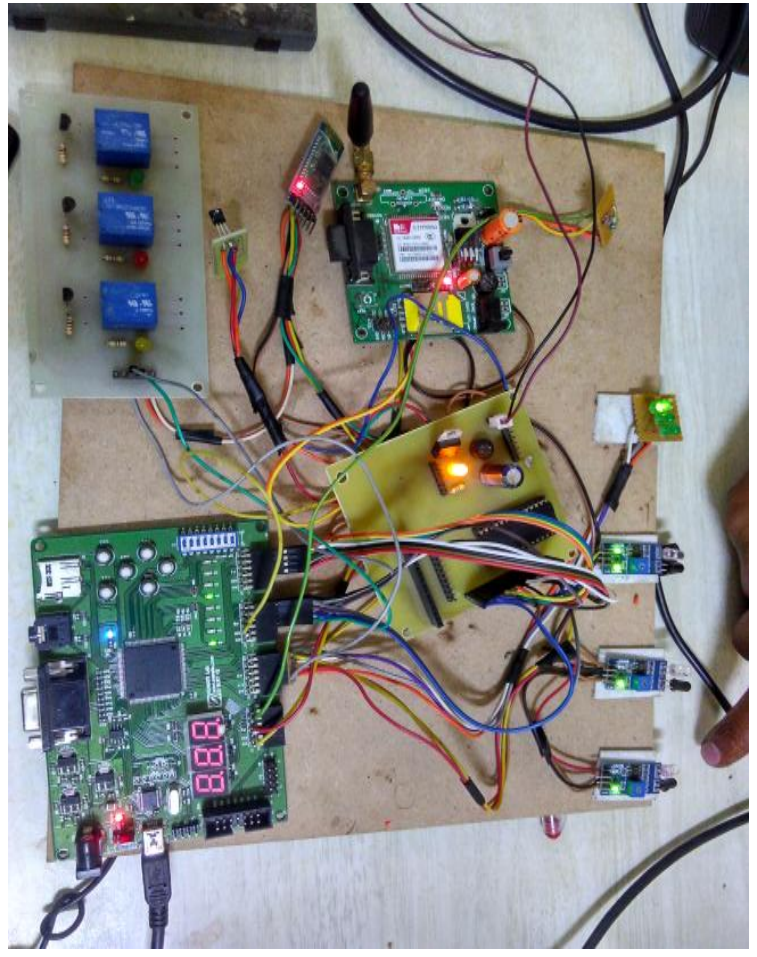

Fig 2: Experimental Setup of the System

\subsection{ADC 809/808}

It is a 8 bit analog to digital converter.It has 8 channel multiplexer and microprocessor compatible control logic.It uses successive approximation technique.It operates on $5 \mathrm{Vdc}, 15 \mathrm{mw}$.Conversion time is $100 \mu \mathrm{s}$.[5]

\subsection{GSM Module (sim 900)}

GSM is a digital communication system which has rapidly gained acceptance and market sheared worldwide. GSM also pioneered low-cost implementation.

\subsection{Bluetooth (HC05)}

Bluetooth is a wireless technology standard for exchanging data over short distances using short wavelength UHF radio waves in the ISM band from $2.4 \mathrm{GHz}$ to $2.485 \mathrm{GHz}$. from fixed and mobile device. A master Bluetooth device can communicate with a maximum of seven devices.[5]

\subsection{Spartan III FPGA Kit.}

Elbert V2 is an easy to use FPGA development board featuring Xilinx Spartan -3A FPGA. This development board features Xilinx XC3S50A chip in TQG 144 package.XC 3S50A family of FPGA solves the challenges in most high volume,cost sensitive, $\mathrm{I} / \mathrm{O}$ intensive electronic applications.Some of its parameters are listed below.

Table 1. XC3S50A Details

\begin{tabular}{|l|l|l|l|l|}
\hline $\begin{array}{l}\text { Device } \\
\text { Name }\end{array}$ & $\begin{array}{l}\text { System } \\
\text { Gates }\end{array}$ & $\begin{array}{l}\text { Eq.Logic } \\
\text { Cells }\end{array}$ & $\begin{array}{l}\text { Distributed } \\
\text { RAM Cells }\end{array}$ & $\begin{array}{l}\text { Block } \\
\text { RAM } \\
\text { Cells }\end{array}$ \\
\hline XC3S50A & $50 \mathrm{~K}$ & 1584 & $11 \mathrm{~K}$ & $54 \mathrm{~K}$ \\
\hline
\end{tabular}


Table 2. XC3S50A Details

\begin{tabular}{|c|c|c|c|c|}
\hline \multirow[t]{2}{*}{$\begin{array}{l}\text { Device } \\
\text { Name }\end{array}$} & $\begin{array}{l}\text { CLB } \\
\text { Arra } \\
\mathbf{y}\end{array}$ & \multicolumn{3}{|l|}{$\begin{array}{l}(1 \mathrm{CLB}= \\
4 \text { Slices })\end{array}$} \\
\hline & Rows & Columns & CLBs & Slices \\
\hline XC3S50A & 16 & 12 & 176 & 704 \\
\hline
\end{tabular}

\section{ABOUT FPGA}

FPGA is an integrated circuit which can be configured by a customer or designer after manufacturing. The configuration of FPGA is described by using HDLs like VHDL or Verilog.We can develop them to form an ASIC.

\subsection{Architecture of FPGA}

\subsubsection{Configuration Logic Blocks}

Configuration logic blocks are built of flexible look up tables.These look up tables are used to implement logic and data.This is achieved by flipflops or latches.

\subsubsection{Input Output Blocks}

IOBs are used to control the flow of data between I/O pins and internal logic of the device.IOBs support bidirectional flow and also tristate operation.

\subsubsection{Block RAM}

It stores data.Its storage capacity is $18 \mathrm{kbits}$.

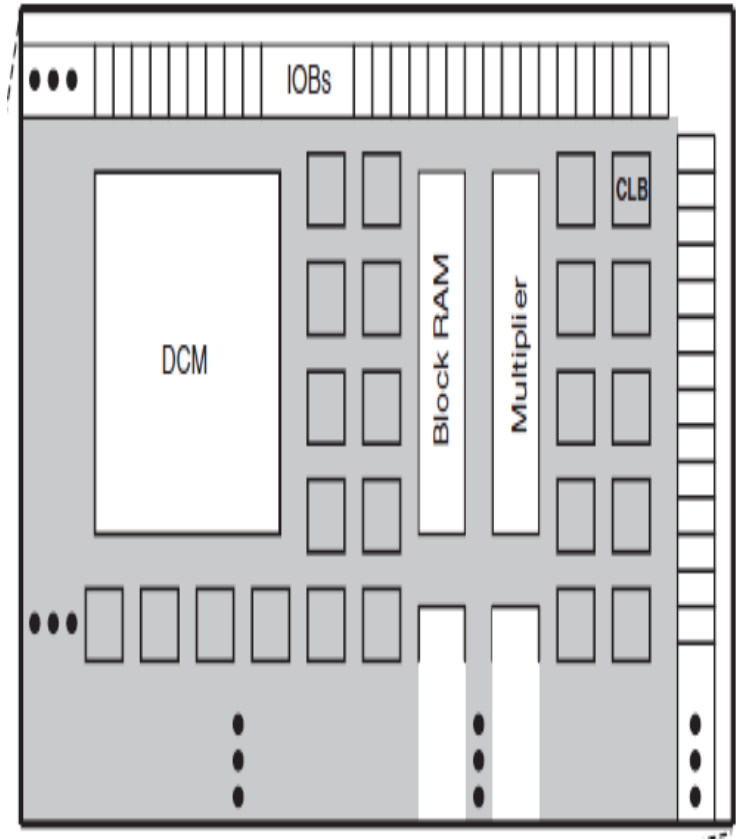

Fig 3: Internal diagram of FPGA (from Xilinx XC3S50A datasheet)

\subsubsection{Multiplier Blocks}

It accepts two 18 bit binary no. as inputs and calculates the product.

\subsubsection{Digital Clock Manager(DCM)}

This block manages the clock signals for distributing,delaying,multiplying,dividing and phaseshifting.It also provides calibration.

FPGAs can be programmed with different tools such as C, $\mathrm{C}++$, SystemC, MyHDL, CHISEL, JHDL, BSV, MATLAB,
Labview FPGA,System Verilog, VHDL, Verilog, Spinal HDL.

Different tools used for programming FPGA(simulators) are active HDL, Inclusive Enterprise Simulator(big 3), ISE simulator, model sim and Questa(big 3), MP sim, pure speed, Quartus II simulator (Q sim), SILOS, Verilog XL, Veritak, Vivaldo simulator, LarcusVerilog, Verilator, Verilog Behavioral Simulator(VBS), Veriwell.

\section{RESULTS}

\subsection{Overall Output}

The proposed system is implemented as a prototype using FPGA Spartan-3 kit.The software tool used for simulating the programme is ISE design suit 14.5.The programming language used for writing the software is Verilog. The total hardware used is given below.The system has been experimentally tested for both industrial automation and streetlight illumination. The details of total hardware used of chip from the available hardware is given below.

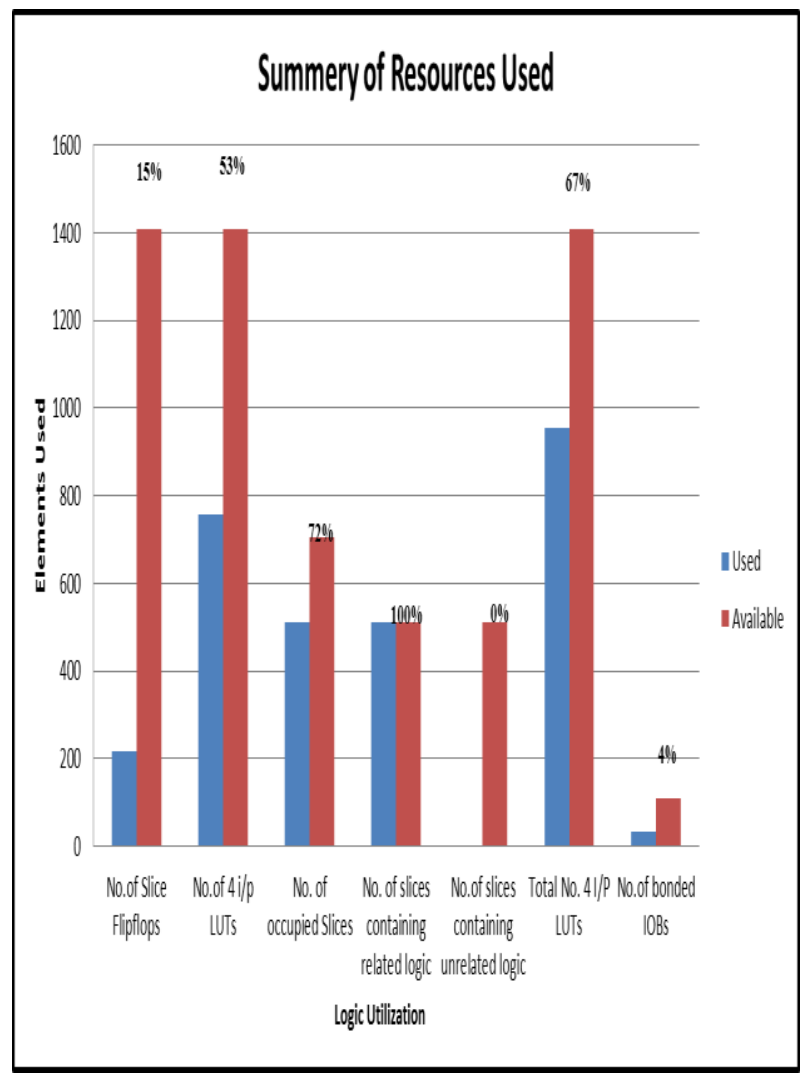

Fig 4: Summary of resources used

\subsection{Simulation Results}

\subsubsection{Generation of clock for ADC}

The clk generated for FPGA is having the clock speed $33 \mathrm{Mhz}$.But the clock required for operating the ADC is scaled down to the required level.For ADC 809 it is scaled down to $1 / 12$ of the crystal frequency 


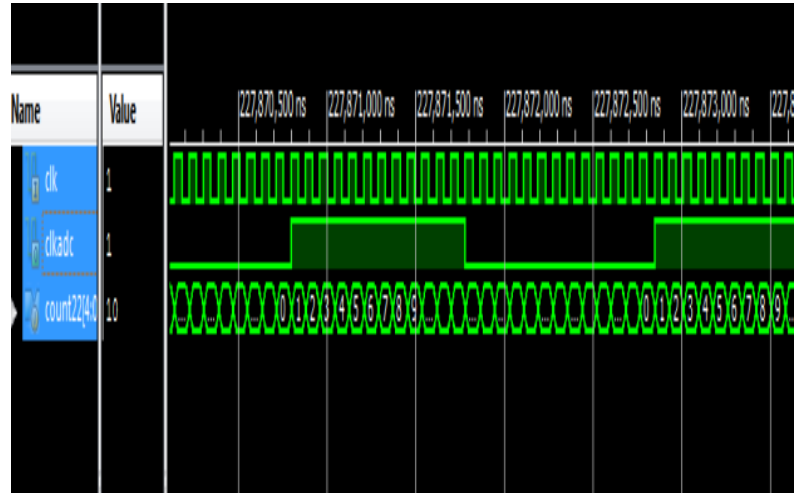

Fig 5: Output of clock generation for ADC

\subsubsection{Output of $A D C$}

The time required for conversion is $100 \mu$ s. After the conversion time elapses EOC (End of Conversion) signal is generated.Active latch enable signal is generated to catch converted data.

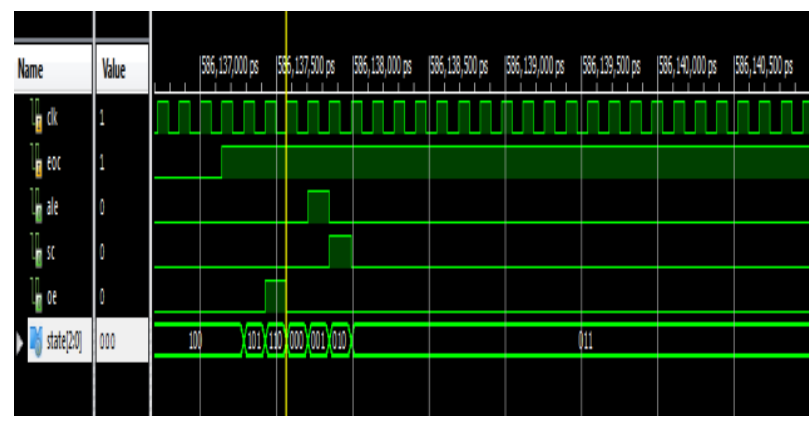

Fig 6: Output of ADC

\subsubsection{Output for driving LEDs and Relays}

After collecting data from different sensors it is given to ADC.It converted in to digital form and stored in its registers.After analyzing data from different registers the relays and accordingly devices connected to made ON/OFF.The output shown also shows the output of LDR and IR sensors and also LED strip connected.Bluetooth is also used to switch ON or OFF the devices.HC05 is used.The messege is passed as a letter A or B or its ascii values such as 41 or 42 .

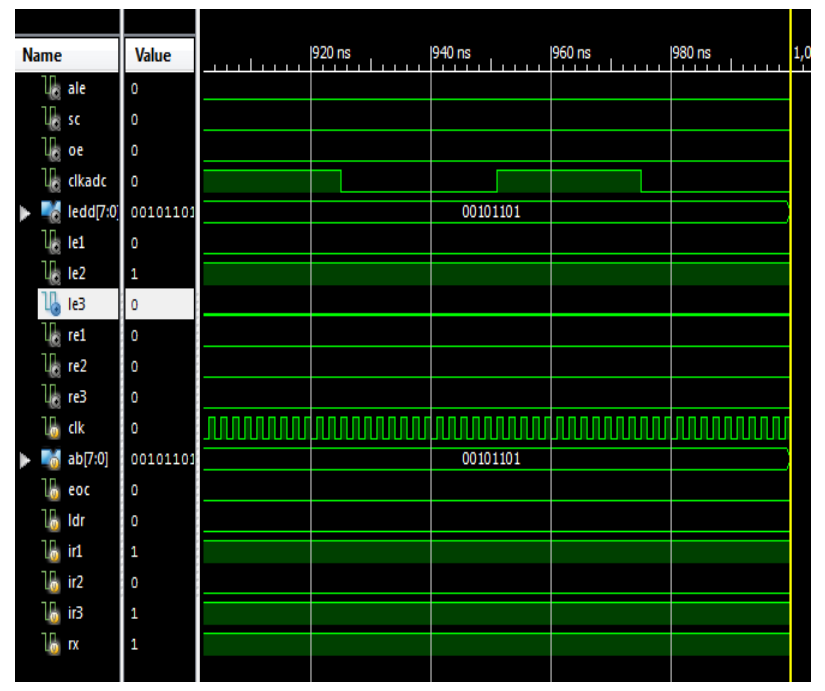

Fig 7: Output of IR Sensors for Glowing LED and for Closing Devices On/Off

\section{CONCLUSION}

In this paper we presented the idea to develop a device which will remotely control the devices in the industry.For the remote operation we are using GSM technology. Also short distance communication is achieved with the help of Bluetooth.It sends the SMS to the operator in case of emergency.Spartan $3 \mathrm{E}$ kit is used for the processing.The software part is written in Verilog.ISE 14.5 suit is used for simulation

\section{ACKNOWLEDGMENTS}

I would like to thank my project guide Prof. Pravin N. Matte for timely help and guiding me to write this paper. I would also like to thank Prof. Rajankumar Bichkar for motivating me to write this paper and presenting the paper in conference.

\section{REFERENCES}

[1] G. Eason, B. Noble, and I. N. Sneddon, "On certain integrals of Lips[1] Dr. K. Babulu, P.S.S.M.Kalyan "Design And Development Of Activation And Monitoring Of Industry Automation SystemVia SMS Through FPGA" www.ijird.com January, 2013 Vol 2 Issue 1

[2] Yadav Vajrangshakti. Ramdayal, K. Srinivasa Rao "An Adaptive Industrial Automation Application Design Using ASIC " IJRRECS/October 2013/Volume-1/Issue6/1093-1099

[3] S.V. Tresa Sangeetha, Dr. S. Ravi and Dr. S. Radha Rammohan "Embedded System Based Industrial Process Automation andRemote Data Logging Using GSM with Web Technology" International Journal of Applied Engineering Research, ISSN 0973-4562, Vol. 8, No. 20 (2013)

[4] Supriya Rai, A. P. Thakare “ Implementation of ReliableWireless Real Time Automation System Based on Android Mobile Phone and FPGA" International Journal of Science and Research (IJSR) ISSN (Online): 2319-7064

[5] N.Chintaiah,K.Rajsekhar,V.Dhanraj "Automated Advanced Industrial and Home Security Using GSM and FPGA " (IJCSIT) International Journal of Computer Science and Info]rmation Technologies, Vol. 2 (4), 2011, 1598-1602

[6] Sridutt Tummalapalli, M V D Prasad "ZIGBEE Operated FPGA Based Nodes in Wireless Industrial Automation Monitoring and Control" International Journal of Engineering Trends and Technology (IJETT) Volume4Issue5- May 2013

[7] Archana M, Mahalahshmi.R "E - Street: LED Powered Intelligent Street Lighting System with Automatic Brightness Adjustment Based On Climatic Conditions and Vehicle Movements" International Journal of Advanced Research in Electrical, Electronics and Instrumentation Engineering Vol. 3, Special Issue 2, April 2014

[8] Deepak Kapgate" Wireless streetlight control system"http://www.researchgate.net/publication/2250078 97

[9] Isah Abdulazeez Watson, Oshomah Abdulai Braimah, Alexander Omoregie" Design and Implementation of an Automatic Street Light Control System" www.ijetae.com (ISSN 2250-2459, ISO 9001:2008 Certified Journal, Volume 5, Issue 3, March 2015) 